\title{
Effect of carboplatin when administered after dacarbazine failure: Clinical benefit of sequential therapy
}

\author{
EVE MERRY $^{1}$, MARIA MARPLES ${ }^{2}$, SARAH DANSON $^{3}$, DAVID HARDING $^{2}$, JAMES DENNY $^{2}$, \\ IGNACIO VAZQUEZ ${ }^{1}$, LOUISE CHESSHIRE ${ }^{2}$, FIONA TAYLOR ${ }^{4}$, ALBERTO FUSI ${ }^{1}$ and ANGUS G. DALGLEISH ${ }^{1}$ \\ ${ }^{1}$ Department of Medical Oncology, St. George's Hospital NHS Trust, London SW17 0QT; \\ ${ }^{2}$ Institute of Oncology, St. James's University Hospital, Leeds LS9 7TF; ${ }^{3}$ Weston Park Cancer Centre, \\ Weston Park Hospital, University of Sheffield, Sheffield S10 2SJ; ${ }^{4}$ Academic Unit of Clinical Oncology, \\ The University of Sheffield, Sheffield S10 2TN, UK
}

Received February 11, 2020; Accepted August 13, 2020

DOI: $10.3892 / \mathrm{mco} .2020 .2144$

\begin{abstract}
Dacarbazine chemotherapy has been the mainstay of melanoma treatment for $>30$ years. In the early $2000 \mathrm{~s}$, carboplatin (with or without other agents, such as paclitaxel) was the most commonly used second-line therapy in the UK. The aim of the present study was to report a significant response rate to second-line carboplatin in patients from three UK institutions who had been previously treated and failed to respond to dacarbazine, and investigate whether sequential therapy may be more effective compared with combination therapy. A total of 104 patients were identified, the majority of whom were treated with carboplatin (area under the curve 5-6) every 3 weeks for a maximum of 6 cycles. A total of 102 patients were evaluable for response, among whom 11 patients had an objective response (1 complete response and 10 partial responses) and 15 had stable disease, giving an overall response rate of $11 \%$ and disease control rate of $26 \%$. The median progression-free survival was 1.8 months (range, 0.2-36+ months) and the median overall survival was 4.6 months (range, 0.2-36+ months). Surprisingly, the majority of the patients who benefited from second-line carboplatin therapy were those with visceral metastases, the survival of whom would not be expected to exceed 6 months after first-line treatment.
\end{abstract}

Correspondence to: Professor Angus G. Dalgleish, Department of Medical Oncology, St. George's Hospital NHS Trust, Blackshaw Road, London SW17 0QT, UK

E-mail: dalgleis@sgul.ac.uk

Abbreviations: PFS, progression-free survival; OS, overall survival; OR, objective response; LDH, lactate dehydrogenase; $\mathrm{CR}$, complete response; $\mathrm{PR}$, partial response; $\mathrm{SD}$, stable disease

Key words: dacarbazine, carboplatin, melanoma, sequential treatment

\section{Introduction}

The outlook for patients with metastatic melanoma has been transformed in the last few years, with $>40 \%$ of patients treated with combination checkpoint inhibitor therapy or combination targeted therapy, in case of BRAF-mutated melanoma, in clinical trials remaining alive after 5 years (1). Novel therapies may hold promise in terms of durable remissions, but the majority of the patients with melanoma experience progression, and some require chemotherapy.

Dacarbazine chemotherapy has been the mainstay of melanoma treatment for $>30$ years. However, the objective response (OR) rate was in the order of 10-20\%, and median survival was not prolonged $(2,3)$, although some patients experienced symptomatic relief, albeit at the cost of limited toxicity. There was no standard second-line therapy, and the recommended approach was clinical trials. Patients who were unable or unwilling to enter a trial, were considered as possible candidates for chemotherapy. In the early 2000s, carboplatin (with or without other agents, such as paclitaxel) was the most commonly used second-line agent in the UK (4). The aim of the present study was to report the response rate to second-line carboplatin in patients with melanoma from three UK institutions who were previously treated and failed to respond to dacarbazine, and examine whether sequential therapy may be more effective compared with combination therapy. This may apply to the incorporation of the newer targeted and immunotherapy treatments available since this study was commenced.

\section{Patients and methods}

Patients and approval. Lists of patients treated with carboplatin for metastatic melanoma were obtained from chemotherapy electronic databases at three tertiary referral cancer centres, namely St. George's Hospital (London), St. James's University Hospital (Leeds) and Weston Park Hospital (Sheffield). The periods covered by the analysis were October 2005-January 2011 for Leeds and Sheffield, and November 2009-September 2015 for St. George's Hospital in London. Permission to perform the analysis was granted by the local committees of all three hospitals. 
Statistical analysis. Demographic disease-related and treatment data were extracted from electronic patient records and entered into an Excel spreadsheet. Data were analysed using Graph Pad Prism software, version 8.0 (GraphPad Software, Inc.).

\section{Results}

Patients and treatment. A total of 104 patients were identified (49 from St. George's Hospital, 35 from St. James's Hospital and 20 from Weston Park Hospital). The patient characteristics are summarised in Table I. The majority of the patients were treated with carboplatin (area under the curve 5-6) every 3 weeks for a maximum of 6 cycles. Carboplatin was administered as second-line treatment after documented disease progression (no planned switch).

Response to treatment. A total of 102 patients were evaluable for response; 11 patients had an OR [complete response (CR), $\mathrm{n}=1$; partial response (PR), $n=10]$, and 15 had stable disease (SD), with an OR of $11 \%$ and disease control rate $(\mathrm{CR}+\mathrm{PR}+\mathrm{SD})$ of $26 \%$. Treatment was generally well-tolerated, with 31 patients requiring at least one dose reduction, and 8 patients discontinuing treatment due to side effects.

Survival. Progression-free-survival (PFS) data were available for all the patients. Overall survival (OS) data were available for 102 patients. All data were censored at 36 months A total of 3 patients remained alive at the time of analysis. The median PFS was 1.8 months (range, 0.2-36+ months) and the median OS was 4.6 months (range, 0.2-36+ months). Patients with an Eastern Cooperative Oncology Group (ECOG) performance status of $0-1$ had a longer PFS (2.1 vs. 1.2 months; $\mathrm{P}=0.0013$ log-rank test; Fig. $1 \mathrm{~A}$ ) and extended median OS (5.0 vs. 2.3 months; $\mathrm{P}=0.0002$; Fig. 1B). Patients with normal lactate dehydrogenase (LDH) levels prior to commencing treatment with carboplatin also had a longer median OS (5.9 vs. 4.5 months; $\mathrm{P}<0.0071$; Fig. 1C). Patients who achieved an OR to treatment had a longer median OS (4.3 vs. 17.5 months; $\mathrm{P}=0.0008$; Fig. 1D) compared with non-responders.

\section{Discussion}

The outlook for patients with metastatic melanoma has improved greatly in the last decade. The anti-CTLA-4 monoclonal antibody ipilimumab was the first drug to achieve prolongation of survival in patients with metastatic melanoma (5). Further improvements have been observed with the introduction of the anti-programmed cell death protein 1 (PD1) antibodies pembrolizumab and nivolumab, which are more effective and less toxic compared with ipilimumab, and with the combination of ipilimumab and nivolumab, which achieved an OR rate of 58\% (6). Patients whose melanomas harbour BRAF mutations also have the option of treatment with BRAF inhibitors (vemurafenib or dabrafenib), or with combinations of BRAF and MEK inhibitors (dabrafenib with trametinib, or encorafenib with binimetinib). The OR with single-agent BRAF inhibitor was $~ 55 \%$, compared with $68 \%$ in patients on combination
Table I. Patient demographics prior to commencing treatment with carboplatin $(n=104)$.

\begin{tabular}{lc} 
Variables & No. \\
\hline Median age, years (range) & $61(23-89)$ \\
Eastern Cooperative Oncology & \\
Group performance status & \\
0 & 48 \\
1 & 28 \\
2 & 13 \\
3 & 3 \\
Unknown & 12 \\
Primary melanoma location & \\
Skin & 91 \\
Mucosal & 2 \\
Ocular & 9 \\
Unknown & 2
\end{tabular}

American Joint Committee on Cancer

stage (version 7)

III

16

IV

Baseline lactate dehydrogenase

Normal

48

Elevated

52

Not recorded

4

First-line regimen

Single-agent dacarbazine $\quad 85$

Temozolomide

Dacarbazine in combination with other agents

Best response to first-line treatment

Complete response 0

Partial response $\quad 12$

Stable disease $\quad 14$

Progressive disease $\quad 76$

Not recorded 2

therapy (7). Over half of the patients treated with combination therapies remained alive at 3 years $(6,7)$.

Despite these advances, it is likely that most patients with metastatic melanoma will relapse. A number of patients are not suitable for combination therapy, others are unable to receive immunotherapy due to pre-existing autoimmune conditions, and $60 \%$ of melanomas are wild-type for BRAF (8). These patients may still require treatment, and it is therefore important to assess pre-existing therapies and evaluate previous experience. To the best of our knowledge, the present review of 104 consecutive patients in three centres who were treated with carboplatin for metastatic melanoma is the largest such series to date. In our cohorts of patients, response and disease stabilisation were observed, with no unexpected complications. Patients with an ECOG performance status of $0-1$, normal levels of LDH, and those who achieved an OR, exhibited a significantly longer OS, in keeping with the data in the literature $(9,10)$. Surprisingly, the majority of the 
A

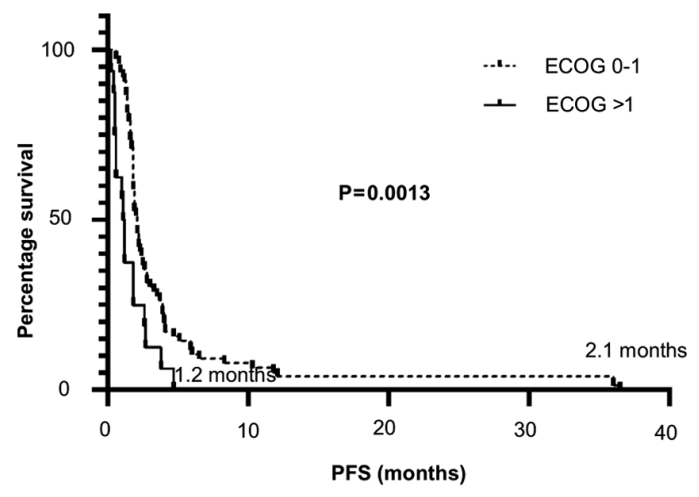

C

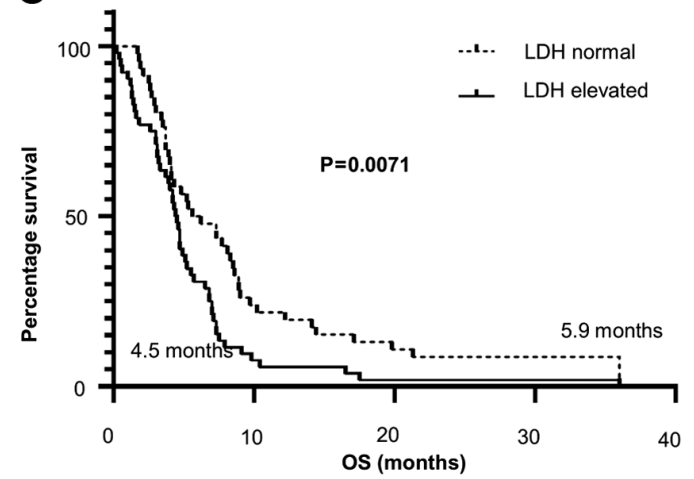

B

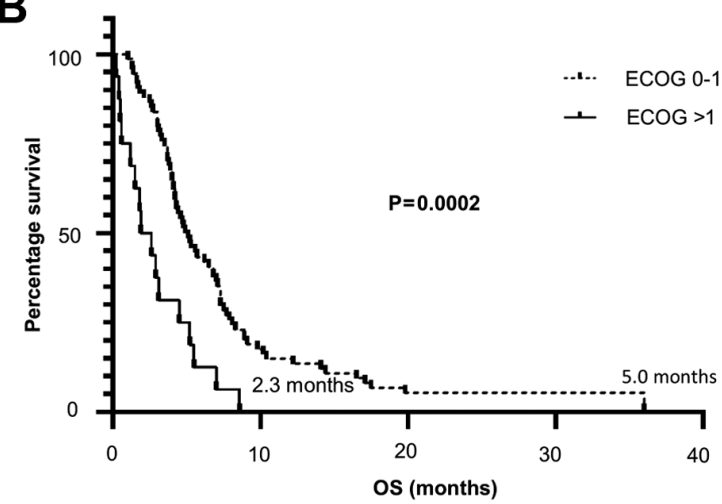

D

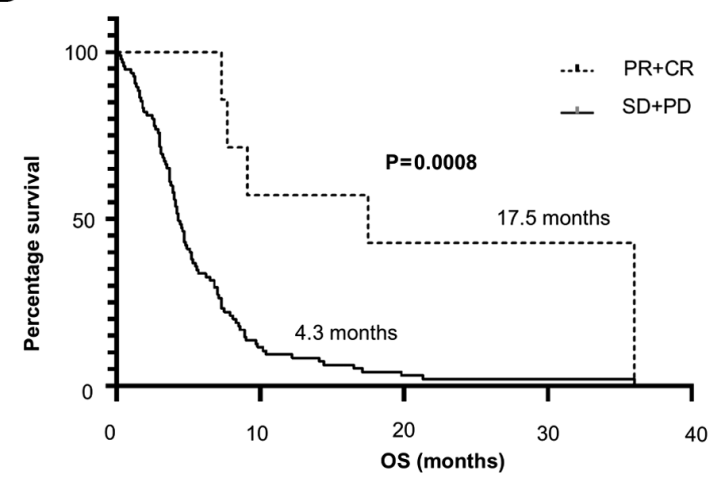

Figure 1. (A and B) PFS and OS in patients treated with carboplatin according to ECOG performance status score (0-1 vs. $>1$ ). OS according to (C) the levels of LDH (normal vs. elevated) and (D) the objective response to treatment (PR + CR vs. SD + PD). OS, overall survival; PFS, progression-free survival; ECOG, Eastern Cooperative Oncology Group; LDH, lactate dehydrogenase; PR, partial response; CR, complete response; D, stable disease; PD, progressive disease.

patients who benefited from second-line carboplatin therapy were those with visceral distant metastases, the survival of whom would not be expected to exceed 6 months after first-line treatment. Dacarbazine plus cisplatin or carboplatin, when given together, appear to have no synergistic benefit (11), and it is unclear whether dacarbazine exerts any significant effect on the susceptibility of tumour cells to carboplatin, or whether there is an indirect effect on the microenvironment or the immune response. It is therefore of great interest that a small but true benefit was observed with the sequential use of these agents, where the overall clinical benefit of $26 \%$ is notably higher compared with that reported by several first-line studies $(2,3)$. It is particularly important, given that there appears to be a concern that multiple drug combinations may achieve better response rates, but do not affect the overall outcome or survival. Of the 3 patients who remain alive and who had an OR to treatment, 2 also received a course of low-dose interleukin-2 post-carboplatin, which may also suggest a type of synergy of the sequential treatment (12).

In conclusion, the observations of the present study may provide a rationale for exploring the potential of carboplatin in patients with failure of targeted treatment and immunotherapy, and for other treatments to be used sequentially rather than concurrently, where the only definitive outcome that can be expected is enhanced toxicity.

\section{Acknowledgements}

Not applicable.

\section{Funding}

The work of AF is funded by the Institute for Cancer Vaccines and Immunotherapy-ICVI (Registered Charity no. 1080343).

\section{Availability of data and materials}

The dataset used and analysed during the present study is available from the corresponding author on reasonable request.

\section{Authors' contributions}

$\mathrm{MM}$ and AGD conceived the original idea for the manuscript. DH, LC, JD, EM, FT, SD and IV collected the data. AF analysed the data. AGD and AF wrote the manuscript. All the authors have read and approved the final manuscript.

\section{Ethics approval and consent to participate}

Permission to perform the analysis was granted by the Hospitals' Local Ethics Committees.

\section{Patient consent for publication}

Not applicable.

\section{Competing interests}

The authors declare that they have no competing interests. 


\section{References}

1. Mario S: Long-term survival outcomes with new treatments for advanced melanoma: Questions still in need of answers (ASCO post) Available from: https://ascopost.com/issues/ october-25-2019/long-term-survival-outcomes-with-newtreatments-for-advanced-melanoma/. Accessed June 30, 2020.

2. Chapman PB, Einhorn LH, Meyers ML, Saxman S, Destro AN, Panageas KS, Begg CB, Agarwala SS, Schuchter LM, Ernstoff MS, et al: Phase III multicenter randomized trial of the Dartmouth regimen versus dacarbazine in patients with metastatic melanoma. J Clin Oncol 17: 2745-2751, 1999.

3. Middleton MR, Grob JJ, Aaronson N, Fierlbeck G, Tilgen W, Seiter S, Gore M, Aamdal S, Cebon J, Coates A, et al: Randomized phase III study of temozolomide versus dacarbazine in the treatment of patients with advanced metastatic malignant melanoma. J Clin Oncol 18: 158-166, 2000.

4. Lorigan P, Marples M, Harries M, Wagstaff J, Dalgleish AG, Osborne R, Maraveyas A, Nicholson S, Davidson N, Wang $\mathrm{Q}$, et al: Treatment patterns, outcomes, and resource utilization of patients with metastatic melanoma in the U.K: The MELODY study. Br J Dermatol 170: 87-95, 2014.

5. Hodi FS, O'Day SJ, McDermott DF, Weber RW, Sosman JA, Haanen JB, Gonzalez R, Robert C, Schadendorf D, Hassel JC, et al: Improved survival with ipilimumab in patients with metastatic melanoma. N Engl J Med 363: 711-723, 2010

6. Wolchok JD, Chiarion-Sileni V, Gonzalez R, Rutkowski P, Grob JJ, Cowey CL, Lao CD, Wagstaff J, Schadendorf D, Ferrucci PF, et al: Overall survival with combined nivolumab and ipilimumab in advanced melanoma. N Engl J Med 377: 1345-1356, 2017
7. Long GV, Stroyakovskiy D, Gogas H, Levchenko E, de Braud F, Larkin J, Garbe C, Jouary T, Hauschild A, Grob JJ, et al: Dabrafenib and trametinib versus dabrafenib and placebo for Val600 BRAF-mutant melanoma: A multicentre, double-blind, phase 3 randomised controlled trial. Lancet 386: 444-451, 2015.

8. Kong BY, Carlino MS and Menzies AM: Biology and treatment of BRAF mutant metastatic melanoma. Melanoma Manag 3: 33-45, 2016.

9. Minor DR, Moore D, Kim C, Kashani Sabet M, Venna SS, Wang W, Boasberg P and O'Day S: Prognostic factors in metastatic melanoma patients treated with biochemotherapy and maintenance immunotherapy. Oncologist 14: 995-1002, 2009.

10. Hauschild A, Larkin J, Ribas A, Dréno B, Flaherty KT Ascierto PA, Lewis KD, McKenna E, Zhu Q, Mun Y and McArthur GA: Modelled prognostic subgroups for survival and treatment outcomes in BRAF V600-Mutated metastatic melanoma: Pooled analysis of 4 randomized clinical trials. JAMA Oncol 4: 1382-1388, 2018.

11. Jungnelius U, Ringborg U, Aamdal S, Mattsson J, Stierner U, Ingvar C, Malmström P, Andersson R, Karlsson M, Willman K, et al: Dacarbazine-vindesine versus dacarbazine-vindesine-cisplatin in disseminated malignant melanoma. A randomised phase III trial. Eur J Cancer 34: 1368-1374, 1998.

12. Legha SS, Ring S, Bedikian A, Plager C, Eton O, Buzaid AC and Papadopulos N: Treatment of metastatic melanoma with combined chemotherapy containing cisplatin, vinblastine and dacarbazine (CVD) and biotherapy using interleukin-2 and interferon-alpha. Ann Oncol 7: 827-835, 1996. 